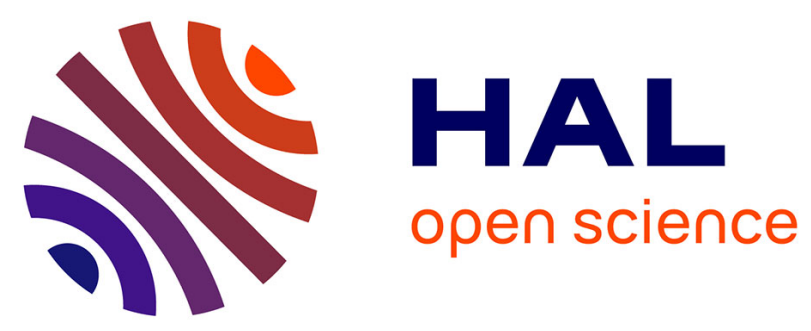

\title{
ULOOF: a User Level Online Offloading Framework for Mobile Edge Computing
}

\author{
José L. D. Neto, Se-Young Yu, Daniel Fernandes Macedo, José-Marcos \\ Nogueira, Rami Langar, Stefano Secci
}

\section{To cite this version:}

José L. D. Neto, Se-Young Yu, Daniel Fernandes Macedo, José-Marcos Nogueira, Rami Langar, et al.. ULOOF: a User Level Online Offloading Framework for Mobile Edge Computing. IEEE Transactions on Mobile Computing, 2018, 17 (11), pp.2660-2674. 10.1109/TMC.2018.2815015 . hal-01547036v2

\section{HAL Id: hal-01547036 \\ https://hal.sorbonne-universite.fr/hal-01547036v2}

Submitted on 14 Mar 2018

HAL is a multi-disciplinary open access archive for the deposit and dissemination of scientific research documents, whether they are published or not. The documents may come from teaching and research institutions in France or abroad, or from public or private research centers.
L'archive ouverte pluridisciplinaire HAL, est destinée au dépôt et à la diffusion de documents scientifiques de niveau recherche, publiés ou non, émanant des établissements d'enseignement et de recherche français ou étrangers, des laboratoires publics ou privés. 


\title{
ULOOF: a User Level Online Offloading Framework for Mobile Edge Computing
}

\author{
José L.D. Neto, Se-young Yu, Daniel F. Macedo, José M.S. Nogueira, Rami Langar, Stefano Secci
}

\begin{abstract}
Mobile devices are equipped with limited processing power and battery charge. A mobile computation offloading framework is a software that provides better user experience in terms of computation time and energy consumption, also taking profit from edge computing facilities. This article presents User-Level Online Offloading Framework (ULOOF), a lightweight and efficient framework for mobile computation offloading. ULOOF is equipped with a decision engine that minimizes remote execution overhead, while not requiring any modification in the device's operating system. By means of real experiments with Android systems and simulations using large-scale data from a major cellular network provider, we show that ULOOF can offload up to $73 \%$ of computations, and improve the execution time by $50 \%$ while at the same time significantly reducing the energy consumption of mobile devices.
\end{abstract}

Index Terms-Computation Offloading, Edge Computing, Android.

\section{INTRODUCTION}

Mobile applications are expanding beyond our day-to-day activity, and mobile computation is becoming more frequent and intense. According to Chaffey's report [1], both the number of users and the time spent using mobile devices exceeded the desktop use. Users spend at least $15 \%$ of their time playing mobile games and another $20 \%$ for entertainment that requires intense computation power and energy.

Mobile devices have limited processing power [2] and battery charge [3] by nature. To overcome these limitations, mobile computation offloading solutions were proposed [4], [5], [6] to delegate intensive computation tasks to more capable computing device(s). With the recent advances in Mobile Edge Computing (MEC) [7], computation offloading gains industrial interest after more than a decade of academic research activities.

Computation offloading supports MEC by adjusting where the computation will take place based on the network and user context. Fig. 1 presents the different characteristics of the networks that a user employs throughout the day. Each network has a different sojourn time (represented by the blue circles), and different associated processing and latency capabilities, due to the use of local operator clouds, private cloudlets (also called MEC hosts) and

- J.L. D. Neto is with Google Inc, Brazil. Email: joseleal@google.com.

- S.Yu is with Northwestern U., USA. Email: young.yu@northwestern.edu

- S. Secci is with Sorbonne Université, CNRS, LIP6. Email: stefano.secci@sorbonne-universite.fr

- D.F. Macedo and J.M.S. Nogueira are with the Univ. Federal de Minas Gerais, Brazil. Emails: \{damacedo, jmarcos\}@dcc.ufmg.br.

- Rami Langar is with LIGM CNRS-UMR 8049, University Paris Est Marnela-Vallee (UPEM), France. Email: rami.langar@u-pem.fr

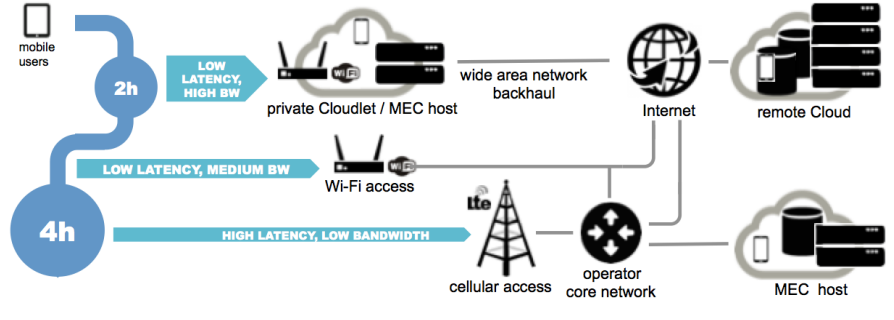

Fig. 1. Mobile computation offloading scenarios

remote clouds. Cloudlet/MEC deployments are expected to favour computation offloading that have not emerged yet with legacy cloud deployments, thanks to their ability to strongly decrease the access latency because of geographical vicinity between users and servers. We assume that at each level the network provider or an over-the-top service provides VMs running an offloading service.

A core element of any mobile computation offloading framework is the decision engine, since it determines when offloading a task to an external (MEC) server counterbalances the related overhead; hence the offloading decision shall be based on predictions of the energy and time required to offload the task, among other possible metrics. It is not trivial to predict the execution time and energy consumption of an application method ahead of its execution. An offloading framework addresses these challenges to make efficient offloading decisions and also to provide application developers and/or users a way to integrate their application into the offloading framework. Most offloading frameworks proposed so far predict the available bandwidth or execution time, as done in CloneCloud, MAUI and COSMOS proposals [8], [5], [9], without considering variable wireless network capacity over time. Another common assumption is that the inputs of the computation do not vary much, as in [5], [10], which can lead to imprecise estimations.

This article presents an offloading framework called UserLevel Online Offloading Framework (ULOOF). It is equipped with novel algorithms aimed to estimate the execution time and energy consumption of application methods, as well as a location-aware wireless network capacity estimator. This article improves the preliminary work described in [11] by a more detailed description and analysis of the framework, an enhanced decision engine logic in particular in the execution time and energy profiling parts, and by conducting a novel set of experiments and simulations. Our contributions can be summarized as follows.

- We designed and developed a comprehensive mobile offloading framework that does not require neither superuser privileges on the mobile device nor modifications to the 
underlying operating system.

- We conceived and developed a decision engine that provides accurate execution time and energy consumption estimations to support the offloading decision, while requiring minimum user effort for the code instrumentation.

- We evaluated our framework both by simulations using real cellular mobility data and by real-world experiments using a proof-of-concept implementation 1

The remainder of the article is organized as follows. Section 2 discusses the state of the art. Section 3 specifies the ULOOF framework. Section 4 describes the decision engine, the problem model and the prediction algorithms. Section 51 describes the tested applications. Section 6 reports simulation results obtained by processing real large-scale mobility data. Section 7 describes experimental results. Section 8 concludes the paper. An appendix provides more details on the energy profiling.

\section{Related Work}

We review relevant works on mobile computation offloading positioning our framework with the state of the art.

Cuervo et al. [5] propose a framework called MAUI, which focuses on energy saving. It uses a profiler that measures energy consumption and a solver that decides whether to offload or not a method based on the measurements provided by the profiler. The authors evaluate MAUI using three mobile applications, revealing that computation offloading not only saves energy, but also that it allows applications to run faster.

Chun et al. [8] propose CloneCloud, which partitions the application binary with a set of execution points. The execution points are determined so that the resulting partitions are executed in the most efficient execution environment. As a result, Clonecloud can determine the most efficient execution points and execution configuration for each partition.

Kemp et al. [4] propose Cuckoo, a simple offloading framework that always offloads a method when the remote server is available. Cuckoo implements a library to manage the communication between the mobile device and a communication middleware.

Verbelen et al. [12] propose AIOLOS, an offloading framework focusing on class-level offloading using an OSGi framework. They provide an Eclipse IDE plugin that helps developers to build an AIOLOS-enabled application bundle in Android. The bundle is executed to update the execution time and to return a size profile. The profile is then used to predict future executions.

Kosta et al. [10] propose ThinkAir. It generates a wrapper for methods to be offloaded so that an execution controller decides whether to offload the method based on execution time, energy and cost. They modelled the execution time and energy using historical data from previous executions. A client handler manages the connection to the remote $\mathrm{VM}$ and is also responsible for managing the $\mathrm{VM}$ configuration.

Shi et al. [9] propose the COSMOS framework; it determines the benefit of offloading based on argument size, upload bandwidth, result size and download bandwidth using a predefined threshold. The predictions are refined at the end of every execution by adjusting the predicted upload and download bandwidth.

Our proposed offloading framework employs a decision engine that can model execution time and energy consumption based on

1. Proof-of-concept applications, using a running server at LIP6, is made available in [26], along with a demo video. historical execution data. Machine (device) execution time and energy consumption are learned and used to predict the method execution behaviour. Our framework also demands minimal intervention to the normal application development, so neither it requires the developer to understand how the framework operates nor needs modifications/privileges on the Android OS.

Our framework is explained in detail in the next section. As a preliminary insight, let us first report via Table 1 how ULOOF is positioned with respect to the above-mentioned existing mobile computation offloading frameworks that were implemented and presented with an empirical evaluation. A cell is marked with " $\checkmark$ " when the corresponding attributes are featured in the framework, otherwise it is marked with " $x$ ". Intrusiveness corresponds to the level of intervention to the application development to permit a given offloading framework to work; it can be either operating system runtime modifications or modifications in the code and/or development strategies such as programming methods into modules. Some works do not have a decision engine (marked with $\boldsymbol{X}$ ), choosing always to offload when possible, while others have their own decision engine to decide the execution platform of a specific code. Other parameters are self-explanatory. According to this analysis, MAUI is the most similar proposal to ULOOF, however we were not able to access its implementation therefore we could not perform quantitative comparisons.

Furthermore, there are other types of works focusing on allocating computation resources for offloading and optimizing their allocation. Esteves et al. [13] allocate computation resources using the capital-budgeting technique, typically used in the field of financial option valuation. Such a technique allows to choose the best remote servers among many, based on an offloading cost (execution time) and a transmission cost (transmission time). Kristensen et al. [14] approach the resource allocation problem by foraging computation resources from different mobile devices. Mobile devices share their available resource and schedule their work based on their available computation resources. ULOOF is inspired by these resource allocation works, employing a method-level code offloading solution that constantly improves its decisions by learning from previous outputs. Our framework provides computation resource information of both mobile devices and nearby cloud servers to the decision engine, hence allocating computation to the most suitable computing element.

One of the challenging parts of developing a framework for mobile computation offloading is to measure energy consumption of a mobile device. Cignetti et al. [15] provide efficient and accurate energy models for specific models of phones. However, it is difficult to calculate the method energy consumption from a user-level point of view [16], [17], i.e., with the limited system rights and APIs given to a standard mobile application. Zhang et al. [18] provide a general power model using device dependent parameters, and measuring each component energy separately. However, this requires a periodic and active monitoring of the components as a background service, which increases energy usage. Miettinen et al. [19] analyze energy consumption usage of mobile devices to find a comparison between radio and CPU, expressing one part as a function of the other. We complement these works with a non intrusive energy profiling methodology explained in the next sections.

Additional research works on mobile computation offloading adopt as evaluation methodology discrete-time or ad-hoc simulators, instead of actual system implementation with empirical evaluation. Mach et al. [33] surveyed 22 offloading algorithms 
TABLE 1

Comparison between state of the art frameworks and the ULOOF framework

\begin{tabular}{|c|c|c|c|c|c|c|}
\hline Name & Intrusiviness & Decision Engine & OS/Language & Energy Model & User-Level & Plug-and-play \\
\hline MAUI [5] & $\overline{\text { Low }}$ & Imprecise prediction & $\overline{\text { Win/C\# }}$ & Online & $\bar{\checkmark}$ & $\bar{\top}$ \\
\hline CloneCloud [8] & Runtime modification & Offline instrumentation & Android/Java & Offline & $x$ & $x$ \\
\hline Cuckoo [4] & Development in AIDL & $x$ & Android/Java & $x$ & $\checkmark$ & $\checkmark$ \\
\hline AIOLOS [12] & Development in OSGi & $\checkmark$ & Android/Java & $x$ & $\checkmark$ & $x$ \\
\hline ThinkAir [10] & Runtime modification & Imprecise prediction & Android/Java & Online & $x$ & $\checkmark$ \\
\hline COSMOS [9] & Code modification & $\checkmark$ & Android/Java & $x$ & $\checkmark$ & $x$ \\
\hline ULOOF & Low & $\checkmark$ & Android/Java & Online & $\checkmark$ & $\checkmark$ \\
\hline Method o & $\begin{array}{l}\begin{array}{c}\text { Mobile } \\
\text { device }\end{array} \\
\end{array}$ & lattorm & & & & $\downarrow$ \\
\hline
\end{tabular}

Fig. 3. Preparation of an offloading-enabled application

such methods are called, it intercepts the call and makes execution time and energy consumption estimations for both local and remote method execution cases. Then, the decision engine chooses whether to execute the method locally or remotely based on the estimation.

- The remote execution platform takes care of the remote execution of the offloadable computation, by means of a connector module. It executes the requested offloading and returns the result to the mobile device.

To enable this instrumentation, an offloading-enabled Android application (or APK) needs to be prepared. Fig. 3 shows the APK preparation process. First, offloadable methods are marked with an explicit annotation ("@OffloadCandidate”). Then the application is compiled with annotations, and a post-compiler creates a modified APK integrating the offloading logic in the application. The ULOOF post-compiler uses the Soot framework [20].

We also developed a remote execution environment using an Android VM to create an execution environment similar to the mobile device. The remote execution environment runs an android-x86 VM [38] with an offloading platform that receives offloading requests from an ULOOFed application. Since ULOOF requests offloading in terms of Java method level and the execution format on Dalvik and ART is identical, our framework is equally compatible with both Dalvik and ART as long as the library on the remote server contains the method to be offloaded, regardless of the Android API level. The library of the application code is provided when the instrumentation of application happens.

To establish a communication between the offloading-enabled application and the remote method execution environment, we used HTTP to transport serialized objects. Because HTTP is a stateless protocol, it is suitable for a remote execution application.

\section{ULOOF General Framework}

ULOOF is a computation offloading framework that offloads method calls in a user application. Each offloading decision is made based on the energy and execution time estimations. Those estimations are updated after every local or remote execution, so that the framework adapts to changes in the environment.

ULOOF does not require changes in the operating system, or special user privileges (i.e., without 'rooting' the device). This allows us to modify the application without requiring additional knowledge on the depending Android libraries, and decreases security risks due to rooting.

Fig. 2 presents a diagram with the key elements of ULOOF:

- In the mobile device, the instrumentation component instruments the candidate methods for offloading. Whenever

\subsection{On the Offloading Granularity}

An offloading decision must be applied at a specific computing granularity. For example, AIOLOS [12] partitions the application on a per-class basis. Others such as CloneCloud and Cuckoo [8], [4], [5] apply the offloading decision at the method level. We choose to run our framework at the method level because class instances may contain both offloadable and non-offloadable methods (e.g., methods that read/write on the mobile phone's storage).

\subsection{Offloadable Method Selection}

In ULOOF, an application method is considered as offloadable if it has an annotation label (e.g., "@OffloadCandidate”). One 
could expect that the best performance could be reached when it is the developer of the application who explicitly specifies which methods could gain from offloading, based on expert knowledge of the code. However, in some cases, it may be unfeasible for a user that is willing to run task offloading for an application to ask developers to deliver a manual annotation of offloadable methods. While allowing manual annotation (which would be the approach taken by application providers), we also explored in [32] the alternative path of automatically selecting methods to mark as offloadable for an arbitrary application on the market, for which it may be unfeasible to solicit developers for manual annotations.

The automated offloadable method selection algorithm in [32] works as follows: it scans the application structure, detecting non-offloadable methods: they are part of the Android system method, or purely internal (i.e., initializer methods), methods accessing hardware specific features or problematic methods or variables. The remaining methods are then further analyzed to check if they call non-offloadable methods and, if so, they are also discarded. Finally, the remaining methods are annotated as offloadable. We report in Fig. 4 the distribution of offloadable vs non-offloadable categories (including also the ratio of methods using non-offloadable classes) for the top-250 Google play applications applying such automated method selection, showing a median of more than $25 \%$ of offloadable methods.

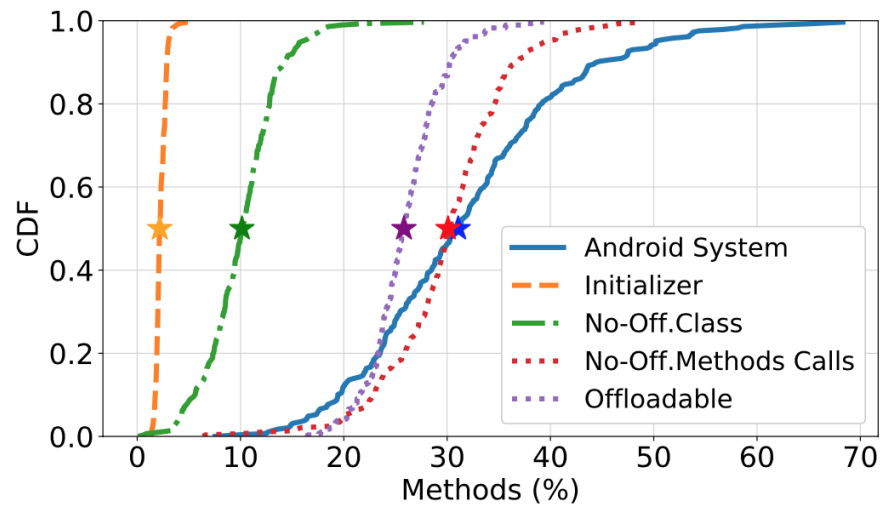

Fig. 4. Offloadable vs non-offloadable methods distribution for top-250 Google Play applications

\section{ULOOF DECISION ENGINE}

This section details the design of the ULOOF decision engine. We expose the design rationale of the framework, execution time and energy consumption prediction algorithms and limitations.

\subsection{Offloading Cost Functions}

The ULOOF decision engine uses cost functions that estimate the energy and time required to run the methods. These cost functions make use of empirical traces to express the cost of running a method in the remote execution application.

Since the offloading problem is a multi-criteria decision problem, we employ a scaling factor, $\alpha$, to prioritize either time or energy in the decision engine. In that way, $\alpha$ can be used to either prioritize saving execution time or battery charge. The equations for the offloading binary decision are:

$$
\begin{aligned}
& L(m)=\alpha \cdot t_{l}(m)+(1-\alpha) \cdot e_{l}(m) \\
& R(m)=\alpha \cdot t_{r}(m)+(1-\alpha) \cdot e_{r}(m)
\end{aligned}
$$

where $M$ is the set of methods of the mobile application, $m \in M$ is the offloading candidate method, $L: M \rightarrow \mathbb{R}$ and $R: M \rightarrow \mathbb{R}$ are the estimated cost functions in case of local and remote execution, respectively. The two components of each cost function are the execution time $(t)$ and consumed energy $(e)$ related to the local or remote execution of method $m$. Lower values for $\alpha$ make the decision tending towards saving energy, while higher values do improve computing responsiveness. Obviously, functions $t$ and $e$ are not working on the same images, i.e., the output ranges are not normalized on the same scale. In this respect, $\alpha$ also works as a normalization factor, and its values may be skewed to either one or zero depending on the $e$ and $t$ images. One may develop an algorithm to adjust $\alpha$ based on the variable network latency, with however a certain impact on the computing overhead and falls outside of the scope of this work. ULOOF triggers method offloading if the following condition holds:

$$
R(m)<L(m)
$$

That is, if the remote execution cost is lower than the local execution cost. We detail how time and energy functions are computed in the following subsections.

\subsection{Execution time prediction}

In ULOOF, annotated methods need to be profiled so that execution time and energy consumption figures can be predicted properly when each method is called to decide whether to offload it or not. There are two possible methodologies to profile the method execution time for computation offloading; either analyzing the target method structure to model the execution time, or predicting it based on the previous execution results and dynamically adjust the prediction at each call. While it is possible to compute a method complexity, e.g., using cyclomatic structural complexity [28], this comes at the risk of big gap with the actual execution time of the method [29]. Hence we follow the latter approach.

In ULOOF, every time an annotated method is called, the execution time and energy consumption for that method are predicted based on the interpolation of historical execution logs. This data is updated upon execution to possibly improve the prediction accuracy at the next execution.

For the very beginning (i.e., after post-compiled application installation), we set a number of initial executions for which there is no prediction made and the offloading decision is taken as a random choice. This number is set to 5 in our tests, for both local and remote executions. Then, after 5 local and 5 remote executions, the decision engine starts using the ULOOF utility functions for taking the offloading decision. To avoid high prediction error in the early executions, the threshold can be increased to any arbitrary number at the risk of causing unnecessary remote executions when the offloading cost is high. The prediction accuracy is expected to increase as the historical execution logs grow, especially after the first executions used for training.

We introduce the concept of 'input assessment' to model the execution time as a function of the method arguments. It converts a list of arguments into a numerical value using an assess(args) function. This function is monotonically increasing with the method time complexity; a higher value of assess indicates that the method should take longer to finish. ULOOF is able to provide input assessment of primitive type arguments, however the application developer may provide its own assessment profiles for their own data structure as an AssessmentConverter interface. The 
prediction of the execution time uses an interpolation approach. We generate an initial curve of the execution time after a few executions (five in our prototype), and each subsequent execution updates the model to improve the prediction accuracy. In order to bootstrap the decision engine, it randomly chooses between local and remote execution until it gathers five executions for each case. The decision also depends on the network state: obviously, if there is no Internet connectivity the decision is not to offload.

To avoid recalculating the curves at each new execution, we use a low-complexity 'lazy' update in our interpolation [21]: a data point from a recent execution does not trigger an update of the entire curve. Instead, only the region of the curve next to that point is updated. We chose the Akima Spline function as interpolator [22] rather than a cubic spline because we found frequent fluctuations through our initial experiments. The Akima spline can maintain value locality and avoid interference of nearby points [23]. One Akima spline series is maintained for local executions and one for remote executions; we refer to them as, $\phi_{l}: N \rightarrow N$ and $\phi_{r}: N \rightarrow N$, respectively.

Therefore, the local execution time $t_{l}(m)$ is defined as follows:

$$
t_{l}(m)=\phi_{l}\left(\operatorname{assess}\left(\operatorname{args}_{m}\right)\right)
$$

Where $\operatorname{args}_{m}$ is the set of arguments from method $m$.

Since ULOOF aims at maximizing the user experience, the remote execution time must account for the network latency due to the uploading of the method arguments and downloading results as well as the running time of the method on the remote platform.

In order to track the remote execution time, the framework gathers the execution time from the server and interpolates the execution time. The network latency depends on the network being used at that time, so latency measurements are stored on a per-network basis. When the mobile device is on Wi-Fi, measurements are bound with the access point $M A C$ address. For cellular networks, the tower unique cell identifier (LAC/CID) is used as the unique identifier. ULOOF also estimates the bandwidth $b$ of each network (as detailed in Section 4.4. Thus, the remote execution time prediction follows:

$$
\begin{gathered}
t_{r}(m)=\phi_{r}\left(\operatorname{assess}\left(\operatorname{args}_{m}\right)\right)+d_{r}(m) \\
d_{r}(m)=\operatorname{size}(m, r) / b
\end{gathered}
$$

Where size $(m, r)$ returns the amount of data required to send the arguments for remote execution and to retrieve the results. Therefore, $d_{r}: M \rightarrow \mathbb{R}$ gives the estimated transfer delay.

\subsection{Energy consumption prediction}

The aim of the ULOOF energy model is to build an energy consumption profile based on the empirical data measured in the device; such a profile could be based on public device-specific records or locally generated measures.

The proposed model does not consider energy consumption from components such as the screen, GPS and file I/O nor offload methods which access these components. This is because offloading methods using these components provide a small benefit [31].

We define the energy consumption for local and remote execution as $e_{l}(m)$ and $e_{r}(m)$, respectively, as follows:

$$
\begin{gathered}
e_{l}(m)=c_{c p u}\left(k_{m}, m\right)+c_{\text {radio }, l}(m) \\
e_{r}(m)=c_{\text {radio }, r}(m)
\end{gathered}
$$

$c_{c p u}: \mathbb{R} \times M \rightarrow \mathbb{R}$ is the execution time component, a function of the method $m$ and its CPU usage $k_{m}$. The CPU usage is expressed in CPU ticks. More precisely, the estimated execution time is computed as follows:

$$
c_{c p u}(m)=l_{\text {cpu }}\left(k_{m} / \text { runtime }_{m}\right) \cdot \text { runtime }_{m}
$$

where runtime $_{m}$ is the local execution time for method $m$. $l_{\text {срu }}$ is an estimated energy consumption function based on the tick frequency (number of ticks divided by the known execution time); it is expressed in watt per second. This quantity is then multiplied by the execution time to estimate the consumption for the whole method. $l_{c p u}$ is method-independent and is device-specific.

$c_{\text {radio }, l}: \mathbb{R} \times M \rightarrow \mathbb{R}$ and $c_{\text {radio }, r}: \mathbb{R} \times M \rightarrow \mathbb{R}$ give the consumption of a given method $m$, when executed locally and remotely, respectively (it is considered also for local executions to account for methods using the network regardless of the offloading procedure). They are computed as:

$$
\begin{aligned}
& c_{\text {radio }, l}(m)=l_{\text {radio }}(\tau) \cdot d_{l}(m) \\
& c_{\text {radio }, r}(m)=l_{\text {radio }}(\tau) \cdot d_{r}(m)
\end{aligned}
$$

where $l_{\text {radio }}$ is the energy consumption in watt per second, which is a function of the radio interface throughput $\tau$. To estimate the overall consumption, $l_{\text {radio }}$ is multiplied by the estimated time spent for transferring data. $d_{l}(m)$ and $d_{r}(m)$ are the time spent for transferring data in a local and remote execution, as explained in the next section. When the method is executed locally, it is:

$$
d_{l}(m)=\operatorname{size}(m, l) / b
$$

where size $(m, l)$ is the amount of network traffic when the method $m$ is executed locally.

$l_{c p u}$ and $l_{\text {radio }}$ are therefore device-specific and methodindependent functions. They characterize the device energy consumption profile. If different radios are used (e.g., Wi-Fi and 4G), specific functions are needed. Those can be calculated using hardware profiling, as explained in the appendix.

\subsection{Network bandwidth estimation}

ULOOF periodically measures available network capacity to improve execution time and energy consumption predictions. The transmission delay depends on the capacity available to the device when an offloadable method is called, therefore measuring accurate network capacity is an important part of the decision engine.

The capacity estimation algorithm performs periodic measurements. In order to account for variations in capacity over time, we apply an Exponentially Weighted Moving Average (EWMA) function with the previous capacity measured to smooth the variation using Equation 13, such a function is used because it was successfully employed to smooth noisy RTT values in [7].

$$
\tau_{t+1}=\tau_{t} \cdot(1-\beta)+\tau \cdot \beta, 0 \leq \beta \leq 1
$$

$\tau_{t}$ is the network capacity that was estimated at time $t, \tau_{t+1}$ is the estimated network capacity the next time, and $\tau$ is an empirically computed value. $\beta$ is used to smooth the noise of the capacity changes between times $t$ and $t+1$. The computation of the empirical capacity $\tau$ varies from Wi-Fi and cellular. On a Wi-Fi network, the network capacity is computed as: $\tau=\frac{d}{\triangle t}$ where $d$ is the size of data transferred over the network and $\Delta t$ is the time to send/receive the data. Because cellular towers serve a much larger area than Wi-Fi access points, the capacity in a cellular network 
may vary significantly based on the location. We use the reversed Shannon-Hartley's theorem to estimate the user's capacity. First, ULOOF derives the estimated network capacity $S$ as follows:

$$
S=\frac{\tau}{\log _{2}(1+S N R)}
$$

Where $S N R$ is the signal-to-noise ratio. As the user moves to a different position within the coverage of the same tower, we compute the new capacity $\tau^{\prime}$ using $S$ stored previously and $S N R^{\prime}$ at the current position. We use the Shannon-Hartley theorem to compute $\tau$ for a new location:

$$
\tau^{\prime}=S \cdot \log _{2}\left(1+S N R^{\prime}\right)
$$

Finally, ULOOF maintains historic capacity data for every network that it encounters. When the device is connected to a Wi-Fi network, we associate the SSID of the Wi-Fi network with the capacity. For cellular networks, $S$ is stored in the location-aware database along with LAC/CID.

\subsection{Limitations of ULOOF predictions}

Before reporting experimental and simulation results, let us point out the limitations of the proposed prediction logic.

First, the device-specific energy consumption is chipset dependent, and should be generated for the desired mobile devices. Our approach is described in the appendix, where Eq. 18 assumes no changing in the type of connectivity between Wi-Fi and Cellular (e.g., due to user mobility, interference phenomena, etc) within a single method execution. If interface changes occur (i.e., vertical handover), the real bandwidth and energy consumption may be different from the predicted value.

Second, the assess function should provide a good estimation on the complexity of the candidate offload method. Developers should be aware of the algorithms employed and how their inputs influence the execution time. However, this may not always be an easy task. For example, most algorithms have an execution time bounded to the argument size (e.g., larger integer numbers or larger vectors). Nonetheless, many algorithms do not follow such assumption. For instance, a method that checks integers for primality: Mersenne numbers (numbers in the form of $2^{n}-1$ ) are much easier to check than ordinary numbers. We could not find in the state of the art any method complexity measure based on the data structures or arguments. We give a detailed assessment of execution time prediction in section 7.2

Third, an ULOOFed application is expected to work better over time, since more empirical data points generates more precise estimations. Conversely, calculations that are run occasionally may suffer from worse predictions. One way to refine the decision is to use crowd sourcing. A server would aggregate the data points for methods in an application from multiple users. This server would periodically upload to the mobile devices the most recent CPU execution curves and possibly the cellular speed estimates.

\section{Tested applications}

For the simulations and experiments, we developed two proof-ofconcept applications.

The first one is a navigation application we refer to as 'CityRoute': it finds the shortest route between two points in a graph using a breath-first search algorithm (problem with a $O(V+E)$ time complexity, where $V$ is the number of vertices and $E$ is the number of edges). In this way, we can follow the time-complexity of the offloaded methods, that for a given source-destination pair is a function of the distance between source and destination. With CityRoute, a city map is represented by an unweighted graph, where vertices are crossroads and edges are streets. For the tests, we generated a graph with 120000 edges using the 'SNAP' dataset [24]. Each batch runs 36 route computations; in each computation, a destination is chosen so that the distance from the source randomly ranges from 19 to 140 edges away. We can so obtain a heterogeneous random set of executions. The CityRoute offloaded method is only one related to the breath-first search algorithm (it loads the input graph and finds the shortest path).

Moreover, we developed a second application that calculates a fixed set of Fibonacci numbers to assess the impact of different trade-off values in the decision-making function; this second application allows indeed an even finer correlation of the result to the time-complexity. Six nested methods can be offloaded to compute the Fibonacci number. We use the Fibonacci application for the analysis in Section 7.4 Even if method-level computation offloading can be applied to a variate set of applications, as we mention in Section 3.2, in the following analysis we restrict our tests to the CityRoute and Fibonacci applications because of the capability to correlate each result to a time complexity figure. We release both applications in [26].

\section{Simulations Using REAL MOBiLE tRACES}

As a preliminary assessment, we run simulations using a cellular mobility data-set obtained in the frame of a collaboration with a major French mobile network operator.

\subsection{Simulation environment}

In the provided data-set, each device is located at the LAC (Local Area Code) level whose coverage ranges from few kilometers to dozens of kilometers of radius depending on the population density. We used France-wide mobile user trajectories with a time-stamped list of traversed LACs in a given day. The obtained trajectories are covered by at least two users to ensure 2-anonymity aggregation. We selected those crossing more than 3 LACs to cover large displacements. In this way, we have 36,450 trajectories for our simulations. For each trajectory, we have a succession of LAC locations with a variable LAC sojourn time.

For our simulations, we emulated for each trajectory a situation in which, for each new LAC position, the CityRoute application (described on Section 77) recalculates a batch of shortest routes for a predefined list of 36 destinations. To emulate the ULOOF decision, the following components had to be computed:

- For the execution times and energy consumption figures, we used empirical values obtained in the testbed tests described in the next Section. Those values are reasonably assumed to be independent of the mobility of the user.

- The RTTs for all LAC location pairs, using a propagation delay directly proportional to three times the euclidean distance (to take into account indirect linkage in wired networks), then adding $40 \mathrm{~ms}$ to each RTT to reproduce bufferbloat phenomena typical of cellular networks [25]. Figure 5 gives the resulting RTT distribution, with a maximum at around $85 \mathrm{~ms}$, based on which we computed the network latency to use for offloading decision as the result of $R T T+D_{t}+D_{s}$, where $D_{t}$ is the transmission delay, and $D_{s}$ is serialization/deserialization delay. $D_{t}$ and 


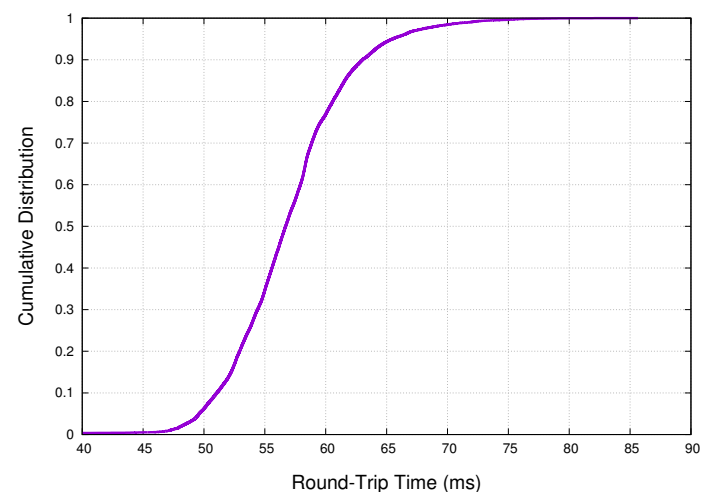

Fig. 5. Round-trip-time distribution from the simulation data-set.

$D_{s}$ are modeled from empirical results obtained in the following evaluation scenarios.

- we used the the utility function trade-off $\alpha$ parameter for equations (1) and (2) set to 0.993 , result of the empirical analysis described on section 7.4

\subsection{Simulation results}

As a first simulation, independent of the individual trajectory, for every possible pair of LAC locations (i.e., any possible pair of user and server locations), we emulated ULOOF decisions using the parameters computed as above explained. The results are given in Figure 6 in terms of execution time and energy consumption, for three cases: with an ULOOF logic, when offloading is not executed ('never offload'), and when offloading always happens ('always offload'). In terms of execution time, there is only a small difference between the 'always offload' case and the ULOOF logic, and that the total execution time can be reduced by a factor between $49 \%$ and $55 \%$ with respect to the 'never offload' legacy approach. Instead, in terms of energy gain, we remark a higher gain granted by the ULOOF logic with respect to the "always offload' approach; moreover, the gain with respect to the 'never offload' case ranges from $40 \%$ to $47 \%$. Overall, these results show that offloading can grant quite significant gains; moreover, the major advantage of using ULOOF instead of an 'always offload' approach appears to be, based on this simulation data, the energy gain rather than the execution time, which somehow confirms what found with the analysis of Fig. 5 and 6.

As a second analysis, we exploit the individual user trajectories and we compute the overall experience for each trajectory. We simulated two configurations for the cloud offloading server:

- Remote cloud: whatever the position of the user is, the cloud facility is always based in one position (i.e., no cloud service mobility associated with user mobility). We fixed this position to a central LAC in Paris.

- Nearby cloud: the cloud facility is fixed in the nearest LAC, hence assuming there is a virtual network overlay managing the network embedding and VM migrations (as envisioned in MEC specifications and investigated in [27]).

Figures 7 and 8 show the obtained results. All the 36,450 trajectories were used here to extract an individual offloading experience assessment. In this analysis, we assumed that when a user changes its LAC, the CityRoute application is executed and the energy and time performance is stored. The weighted average of each performance result (time or energy gain) is then computed,

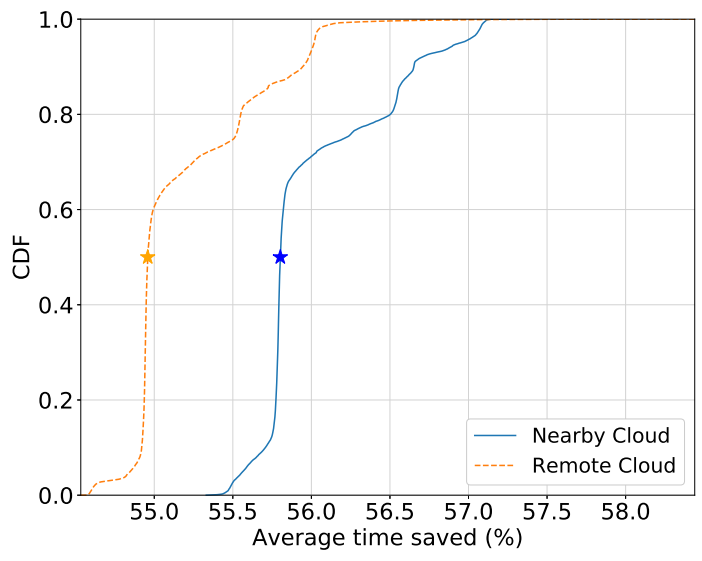

Fig. 7. Execution time gain cumulative distribution (simulation).

weighting each offloading result with the sojourn time of the user in the given LAC. We calculate the weighted average $W_{a}$ as:

$$
W_{a}=\frac{\sum_{i}^{k} g_{i} t_{i}}{\sum_{i}^{k} t_{i}}
$$

where $i$ is a trajectory of a user with the maximum $k$ trajectories, $g_{i}$ is the resource gain of a user in the trajectory $i$ and $t_{i}$ is a sojourn time of a user at the trajectory.

The results show that the median of the execution time gain is 55\% for the remote cloud case and $55.8 \%$ for the nearby cloud case. In terms of energy gain, the median gain ranges from $49.3 \%$ to $49.7 \%$, roughly, respectively. The remote execution consumes less energy because the offloading decision in the simulation is skewed towards shortening the running time. A small part of the methods run in the nearby cloud actually consume more energy than in the mobile device, however they are run in the cloud in order to reduce the response time. Those methods are not run in the remote cloud because the RTT is longer, and hence there is no benefit, either in energy or in runtime, of a remote execution.

As the remote cloud has longer RTT compared to the nearby cloud, it consumed less energy as shown in Fig 6 ULOOF did not offload a small percentage of the methods that consume more energy when offloaded because the RTT is longer, and hence there is no benefit, either in energy or in runtime, of a remote execution.

With respect to the trajectory-agnostic results in Fig. 6 we can notice that using a nearby cloud does not lead to visible gains, with minor differences for both execution time and energy consumption. This seems to suggest that running the service in a fixed VM in a central location of the access network does not lead to significantly lower performance than a case where the VM is moved closer to the user in a large-scale network.

\section{EXPERIMENTAL RESULTS}

We developed a proof-of-concept navigation application we refer to as 'CityRoute': it finds the shortest route between two points in a graph using a breath-first search algorithm (problem with a $O(V+E)$ time complexity, where $V$ is the number of vertices and $E$ is the number of edges). In this way, we can follow the time-complexity of the offloaded methods, that for a given sourcedestination pair is a function of the distance between source and destination. With CityRoute, a city map is represented by an unweighted graph, where vertices are crossroads and edges are streets. For the tests, we generated a graph with 120000 edges using the 'SNAP' dataset [24]. Each batch is composed of 36 


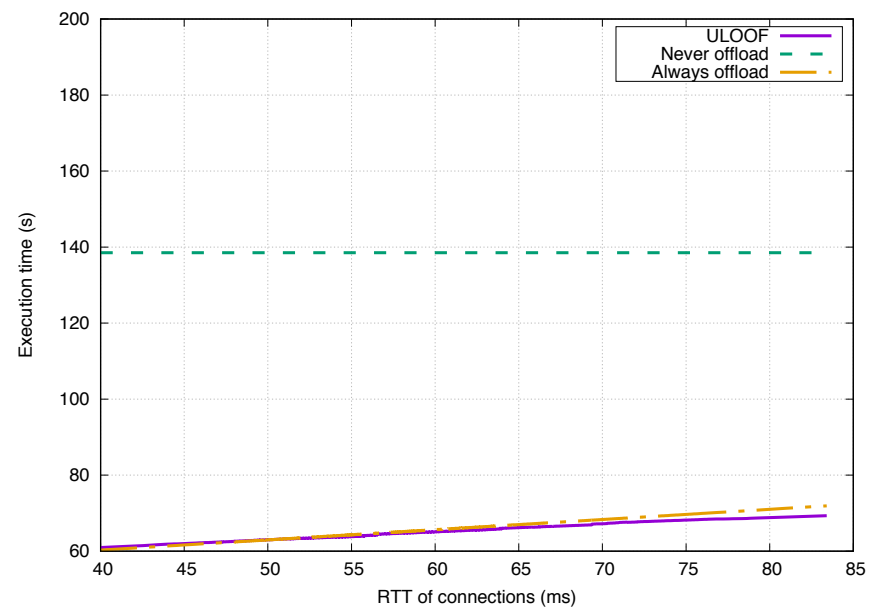

(a) Execution time

Fig. 6. Performance as a function of the RTT (simulation).

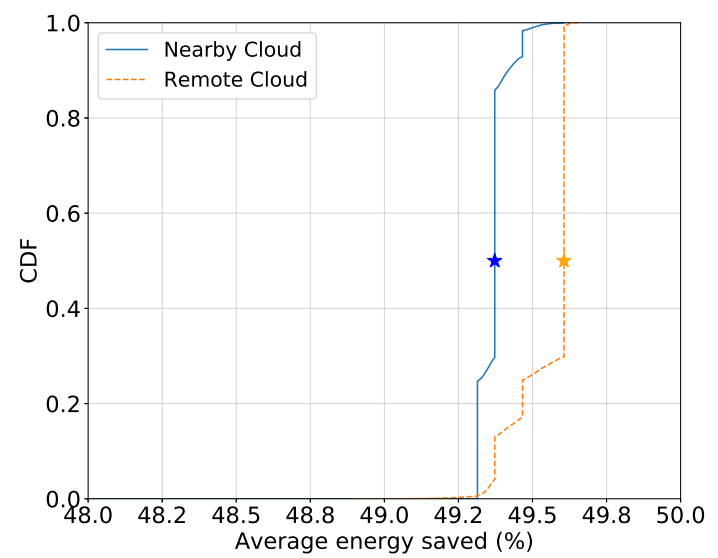

Fig. 8. Energy gain cumulative distribution (simulation).

route computations; in each computation, a destination is chosen so that the distance from the source randomly ranges from 19 to 140 edges away. In this way, we can obtain a heterogeneous random set of executions. The CityRoute offloaded method is only one related to the breath-first search algorithm that loads the input graph and finds the shortest path. We use such realistic application for the results in the following in Sections 7.1 and 7.3

Moreover, we developed a second application that calculates a fixed set of Fibonacci numbers to assess the impact of different trade-off values in the decision-making function; this second application allows indeed an even finer correlation of the result to the time-complexity. Six nested methods can be offloaded to compute the Fibonacci number. We use the Fibonacci application for the analysis in Section 7.4 Even if method-level computation offloading can be applied to a variate set of applications, as we mention in Section 3.2 in the following analysis we restrict our experiments to the CityRoute and Fibonacci applications because of the capability to correlate each result to a time complexity figure. We release both applications in [26].

We consider three different usages of the CityRoute application: an unmodified application, an ULOOFed application with $\alpha$ set to 0 (i.e., energy driven), and another case with $\alpha$ set to 1 (i.e., time driven) to compare the execution time and energy consumption of each case. A desired $\alpha$ value can be computed as in Section 7.4. We used a Samsung Galaxy S5 with a Snapdragon

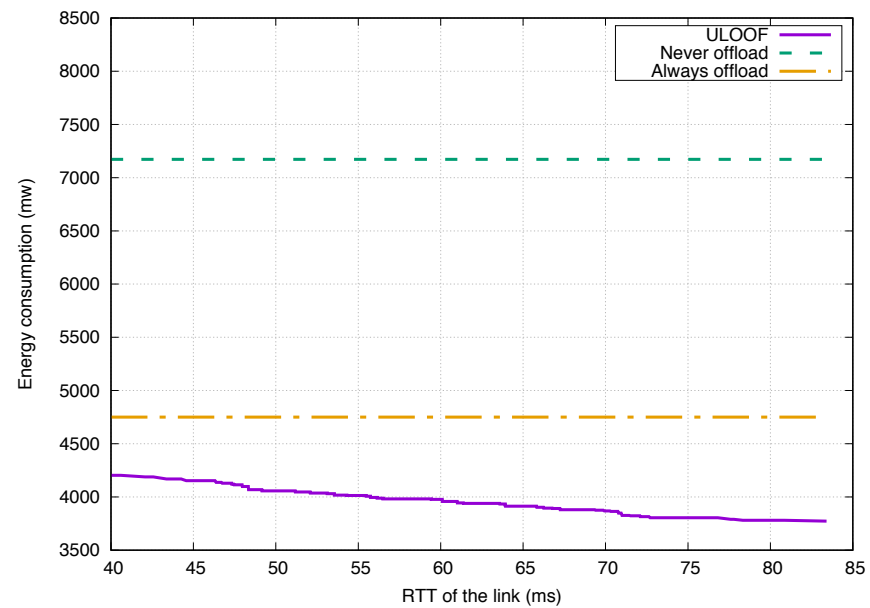

(b) Energy consumption

801 processor, a $2.5 \mathrm{GHz}$ quad-core CPU and 2GB RAM as a mobile device in our experiment. The energy and CPU consumption curves were defined empirically with hardware profiling, as detailed in the appendix. An HTTP server runs on Android-x86 [38] to serve remote execution requests from the mobile device.

We set up two scenarii to study the effect of different latencies on the ULOOF performance (Fig. 9 reports the experienced bandwidth for the experiments of the two scenarii):

- Wi-Fi scenario: it is a semi-controlled environment, where the mobile device uses a Wi-Fi network to reach a server located within the same local area network. This is the case for cloudlet/MEC environments envisioned for access networks, hence for simplicity we refer to it as cloudlet use-case. There is no additional latency injected in the Wi-Fi network and it shows less than $1 \mathrm{~ms}$ RTT between the mobile device and the remote server. The server is a VM with a 64-bit GNU/Linux 4.4, running on an Intel i7$4500 \mathrm{U}$ processor with $41.80 \mathrm{GHz}$ cores and 8GB memory.

- Cellular scenario: it is a mobile environment, where the latency with the remote server is higher than in the $\mathrm{Wi}$ Fi case and can vary due to mobility. We used real measurements, with long latencies typically experienced for cellular networks [39]: using a moving vehicle around Belo Horizonte, Brazil, along a predefined route, we measured the network capacity, execution time and energy consumption of the CityRoute application, using as remote server a DigitalOcean VM, in New York, USA.

\subsection{Execution time and energy consumption}

We measured the execution time and the energy consumption of the mobile device while the application is run. We measured the start time and end time of each test, and the battery level during each test (using the Android BatteryManager API).

\subsubsection{Wi-Fi scenario}

Fig. 10 reports the execution time of 20 contiguous CityRoute batches in the horizontal axis, i.e., the last point of each line is the global execution time. During such executions, we noted the instant when the battery drained by $1 \%$ (it is the minimum measurement step available with user-space Android primitives) and 


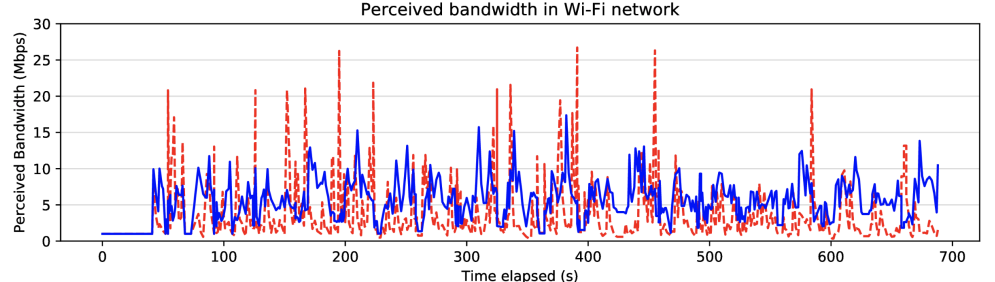

Perceived bandwidth in Cellular network

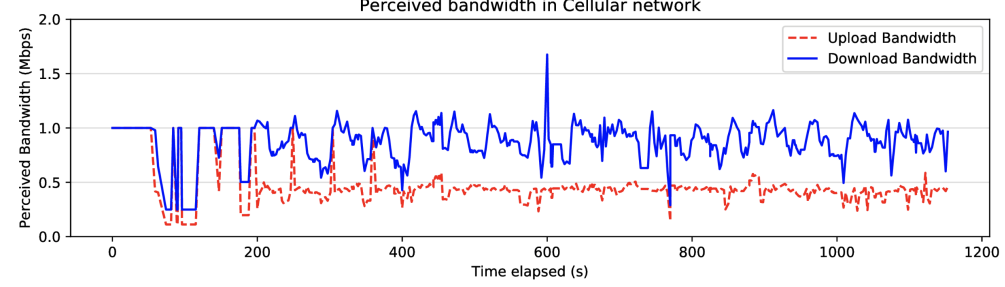

Fig. 9. Change in perceived bandwidth over elapsed time.

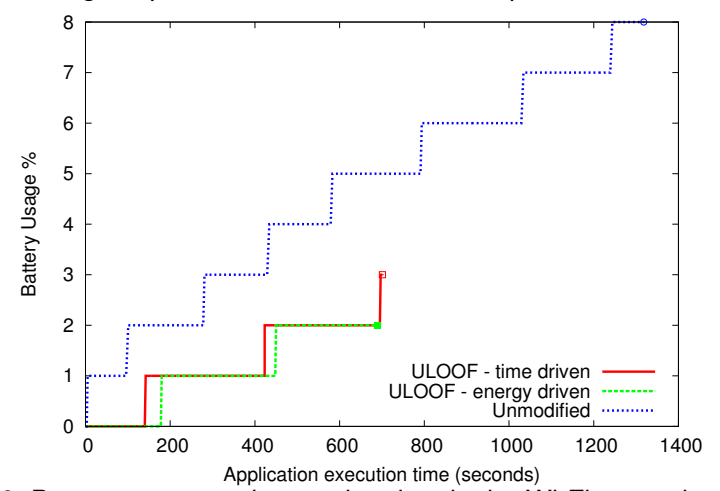

Fig. 10. Battery usage and execution time in the Wi-Fi scenario.

report it accordingly as a vertical step in the figure. Execution time for both ULOOF versions are significantly reduced compared to the unmodified application as noticed from the shorter horizontal length of the plot. Battery usage from both versions is also reduced as shown in the shorter vertical height of plots.

The results show that ULOOF reduced the execution time as well as energy consumption, and that for both time and energy driven variants. More precisely, methods were offloaded $72.95 \%$ and $62.41 \%$ of the times for the time-driven and energy-driven modes, respectively. The execution time was reduced by about $50 \%$. The battery gain ranges from 5 to $6 \%$ in terms of absolute battery consumption, which roughly corresponds to 56 to 224 $\mathrm{mAh}$ for the given phone. The differences in time-driven and energy-driven modes are relatively small compared to the total running time, and oddly the time-driven algorithm took longer to finish compared to the energy-driven mode, which is likely caused by uncontrolled environment variables (e.g. operating system scheduler, background processes, screen state) in the experiments.

\subsubsection{Cellular scenario}

For the cellular scenario, we experimented on a moving vehicle around the city of Belo Horizonte, Brazil, along a predefined route. We first measured the performance of cell towers in the city and then used the data gathered from the measurement.

Fig. 11] shows the accumulated execution times and battery consumed by the CityRoute application executed 20 times in a row. Both time-driven and energy-driven ULOOF improved the execution time and energy consumption compared to the unmodified application. However the absolute gain in execution time and energy consumption has reduced compared to the Wi-Fi/lowerlatency case. More precisely, the decision engine offloaded $27.6 \%$

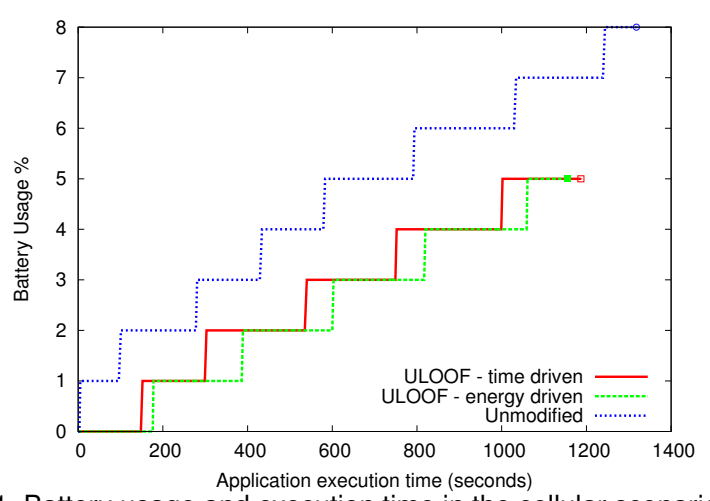

Fig. 11. Battery usage and execution time in the cellular scenario.

of the executions with energy-driven ULOOF and $29.13 \%$ with time-driven ULOOF, with only a small difference between the two modes. The average execution time of the offloaded execution was around 2.5 seconds, above the global average of 1.5 seconds.

Compared to the Wi-Fi scenario, there was $47.64 \%$ longer execution time and $80 \%$ higher energy consumption; this is due to the smaller number of offloads in the higher-latency network. Because of the longer latency, the remote execution time estimate increases and the decision engine decides to offload less often, with more local executions, consuming more energy.

Moreover, the time-driven ULOOF in the higher-latency network had an execution time slightly longer (globally 30 seconds longer, i.e. $2.6 \%$ ) than the energy-driven ULOOF: this is also due to the uncontrolled environment with network latency variations.

\subsection{Prediction accuracy}

To assess the accuracy of ULOOF predictions, we post-processed the execution time and energy consumption for both scenarios. Precisely, we executed the CityRoute application with both local and remote time execution every time the offloadable method is being called; then, we compared the actual running time of these executions with the prediction the decision engine had made.

Fig. 12 reports the prediction error ratio and the average execution time of offloadable methods in terms of the distance between source and destination. The plots are divided into local and remote executions in Wi-Fi and Cellular network, hence each set of plots shows the prediction accuracy of specific network and offloading decision; e.g., the top leftmost plot shows the prediction error of local execution time in Wi-Fi network.

In each graph, the red line on top represents the average execution time with a $95 \%$ confidence interval. The horizontal axis indicates the distance to destination in number of edges in the graph, which for the breadth-first search shortest route computation is an index of the experienced time complexity.

The boxplot reports the relative prediction error of that specific network and offloading decision, with the minimum, first quantile, median, third quantile, maximum of the prediction error, for the specific distance referenced by the horizontal axis. The prediction error is calculated as the difference between the predicted time and the actual time (resp. for the energy consumption).

Each figure block shows results for both local (top) and remote executions (bottom). We had to rely on our energy consumption fitting model for both prediction and the actual consumption. This is because it is not possible to record the energy consumption of a method-level granularity from the device.

We discuss the results for the lower and higher network latency cases in the following sections. It is worth stressing that 

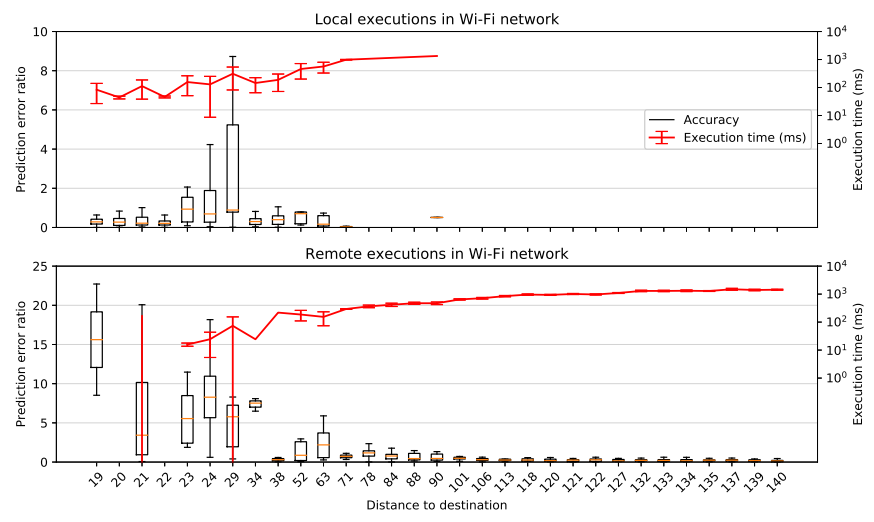

(a) Execution time - Wi-Fi network

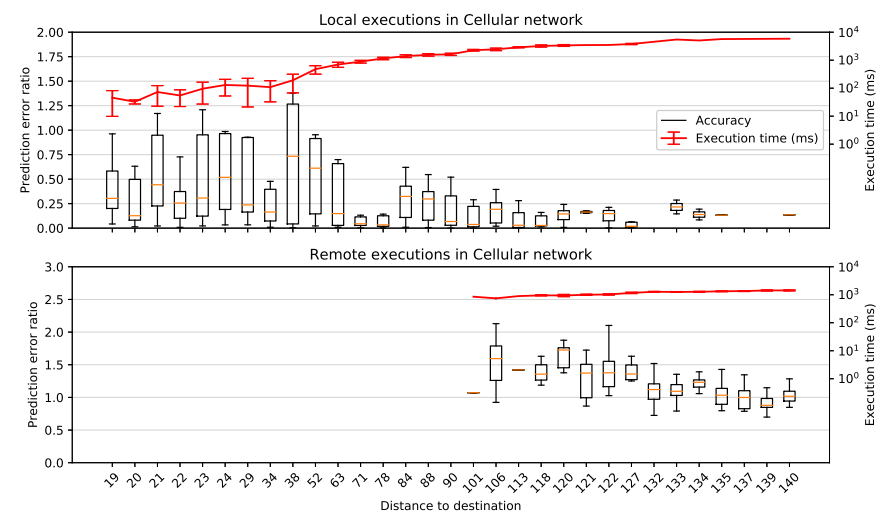

(c) Execution time - Cellular network
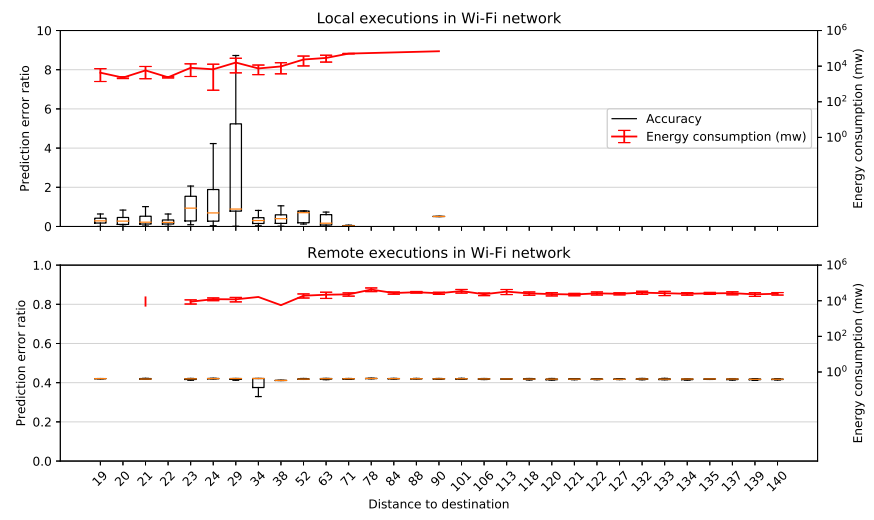

(b) Energy consumption - Wi-Fi network

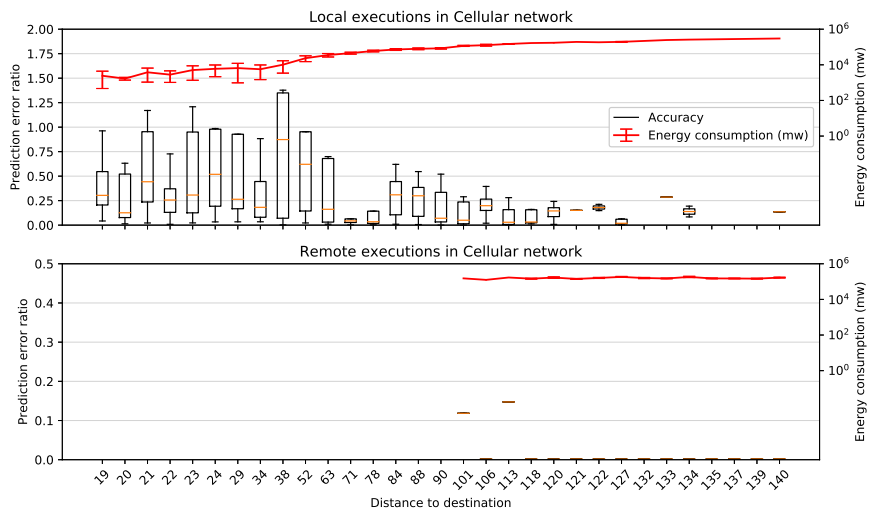

(d) Energy consumption - Cellular network

Fig. 12. Prediction error as a function of method complexity (expressed in ho distance from source to destination for navigation map app).

the samples for the remote execution are concentrated at longer distances because offloading happens less often in executions that take less time. Conversely, the number of samples is lower for local executions at high distances.

\subsubsection{Wi-Fi network}

For the Wi-Fi network scenario, method calls with lower computation execution time suffer from high prediction errors, especially for remote executions. As the execution time increases, the prediction error decreases, with a median always below 50\% starting by 63 hops for local executions. As the processor in the mobile device is shared among processes, the error margin is higher for methods with short execution time.

We can further notice that for the local executions, there is an increasing trend in terms of accuracy as the execution time increases. High errors happen mostly with very low execution times, hence making them less perceivable by the user. For instance, we found there is an average margin of the prediction error of $93.54 \%$ when the computation takes $156 \mathrm{~ms}$ to complete, which decreases to $3.16 \%$ as the execution time increases to 985 ms. For the remote executions, we have a similar trend, with an average margin of the prediction error of $555 \%$ when the execution time is $15.79 \mathrm{~ms}$, which decreases to $14.08 \%$ as the execution time increases to $1435 \mathrm{~ms}$.

The effect of large error margins however does not impact the overall performance of ULOOF. This happens because the offloading will occur only for larger instances of the problem, in which the execution time is much longer. For shorter execution times, most executions happen locally due to the delay required to send data to the remote environment.

The prediction error ratios in energy consumption show that our framework is more accurate when predicting the energy required for remote execution. This happens because the remote execution energy consumption relies heavily on the number of bytes transferred across the execution (i.e. size of argument and result transferred), and the amount of bytes transferred does not differ much for each method call. In contrast, local executions suffer from high prediction errors when the complexity is low, because of the noise related to background computations.

\subsubsection{Cellular network}

Fig. 12(c) and 12(d) show the prediction error in the cellular/higher-latency network experiments.

The prediction error ratio in execution time decreases with higher computation complexity for local executions. For remote executions, instead, the error ratio shows a median between $100 \%$ and $150 \%$, which is likely due to bandwidth variations in the cellular network. Compared to the Wi-Fi scenario, the error ratio is smaller in Wi-Fi because there is less network capacity variation.

The energy consumption prediction is also more accurate for the higher-latency case. As the complexity increases in local executions, the prediction accuracy improves. Because the local execution time and the local energy consumption are closely related (i.e. longer execution consumes more energy) and they both use the Akima interpolation [22], their accuracy is similar. 
For the remote execution, however, the prediction of the energy consumption improves significantly.

\subsection{System overhead}

In terms of system performance, it is important to qualify the overhead caused by the ULOOFed applications. We have measured the overhead of the ULOOF framework when running the CityRoute application by measuring the time difference between the instant when the offloadable method is called and the instant when the decision engine finished the prediction, positioning it with respect to the overall execution time. Fig. 13 shows the time taken for making offloading decision relative to the actual method execution using CityRoute in the cellular network. We measured the overhead of our framework by measuring the time taken to predict execution time and energy consumption against the total method execution time.

The overhead incurred from making offloading decision was less than $40 \mathrm{~ms}$ at all times. For short execution times, the overhead tops at $32 \%$, which is $33 \mathrm{~ms}$ of overhead. However, for longer execution times this overhead is lower than $10 \%$. Although this overhead may be significant for methods that run the least, it is worth noticing that the average execution time is $513.47 \mathrm{~ms}$.

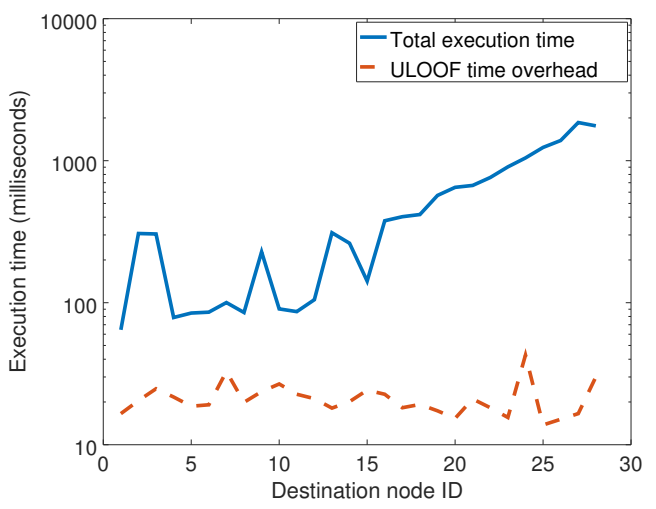

Fig. 13. Overhead caused by the offloading framework.

\subsection{Offloading decision trade-off evaluation}

The $\alpha$ parameter defines how to prioritize between execution time and energy consumption when making an offloading decision. In this, we show the impact of $\alpha$ on the offloading performance. These experiments were performed in a Wi-Fi network.

Besides the CityRoute application, we also use a Fibonacci application computing the Fibonacci number of a random number. In the following analysis we compare three scenarios: 'Always Offload', 'Never Offload', and 'ULOOF' with different values of $\alpha$. Let us recall that $\alpha=0$ means ULOOF considers energy saving only, and $\alpha=1$ for saving execution time, while intermediate values give different trade-offs between these two objectives.

Fig. 14 shows the results for the CityRoute application. The 'Never Offload'curve presents an application that runs all the computation locally, while the 'Always Offload'curve shows the results for the computations always being performed in the remote server. All plots have the horizontal $\alpha$ axis cut to the region where it changes of shape, in this case from 0.98 to 1 . Before that ULOOF does not change its decision because of the unnormalised values for time and energy. In terms of execution time, ULOOF always performs better than the other two scenarios with any $\alpha$ value. The sweet spot is where both lines from the always offloading scenario and ULOOF cross in the energy plot: $\alpha=0.993$.

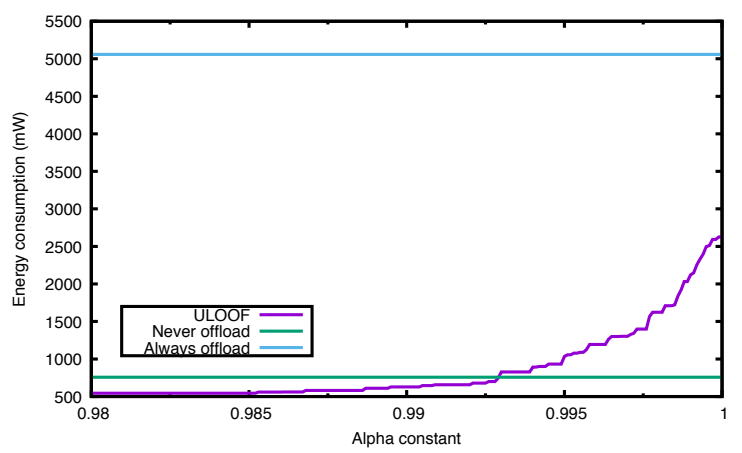

(a) Energy consumption

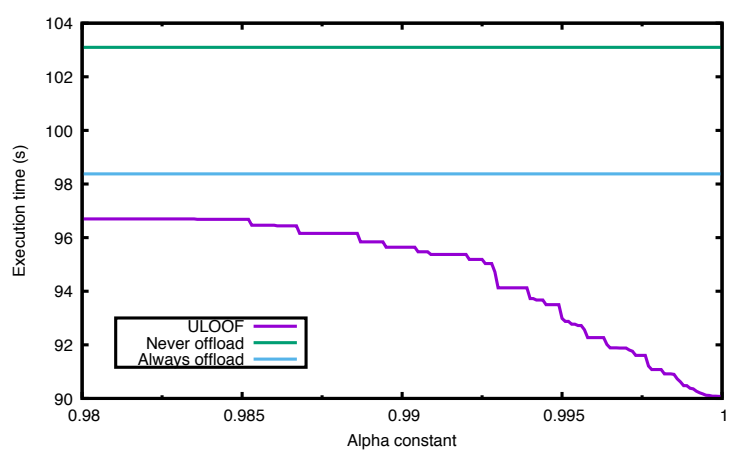

(b) Execution time

Fig. 14. ULOOF performance as a function of $\alpha$ (CityRoute).

In order to show that the ideal value of $\alpha$ depends on the application, the same test was performed using the Fibonacci application. This application demands a meager amount of network, however it is CPU intensive. Fig. 15 shows the results. In this case ULOOF sits in between the two other cases in terms or execution time, and it is always better in terms of both energy consumption and execution time when $\alpha>0.995$.

The last sensitivity test evaluated the effect of transferring larger amounts of data when offloading. This was performed using a modified version of the Fibonacci application, where we transfer a large argument to the offloading method. This forces the large size of argument to be transferred through the network each time the method is to be offloaded. The computation ignores this argument as it is only to increase the transfer size. Fig. 15] and 16 reflect how this changes the performance of the ULOOF framework. The overhead of transferring the large argument impacts greatly the execution time plot, performing time-wise worse than the never offload scenario when favouring energy but for $\alpha>0.99$ for which it has close performance to it. This happens because of the transmission delay involved in transferring the arguments.

\subsection{Comparison between different devices}

To assess the computing performance of different mobile devices used in our experiments, we evaluated the average execution time and energy consumption for a single CityRoute batch. We compare the most recent device available to us, a Samsung Galaxy S7, to a Note 3 and an S5. To avoid bias due to remote execution and focus on system comparison, we executed the CityRoute application with local execution only. Table 2 shows the performance difference between the three devices in terms of CPU usage, as execution time and energy consumption. We report the available live memory as well. The Galaxy Note 3 runs with $30 \%$ lower number of CPU ticks, resulting $41 \%$ faster and $36 \%$ more energy 


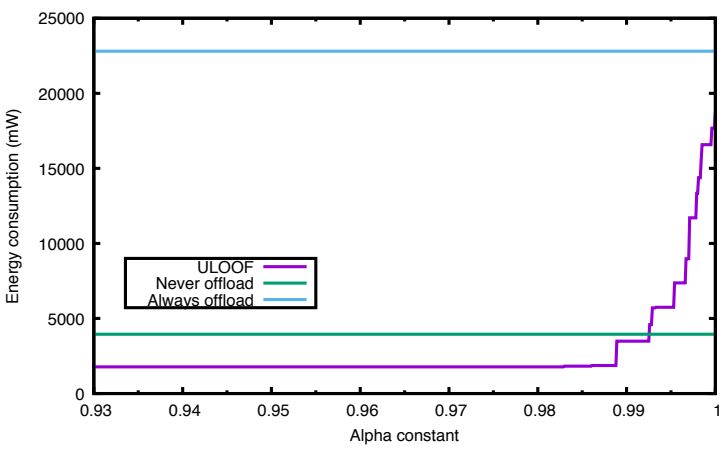

(a) Energy consumption

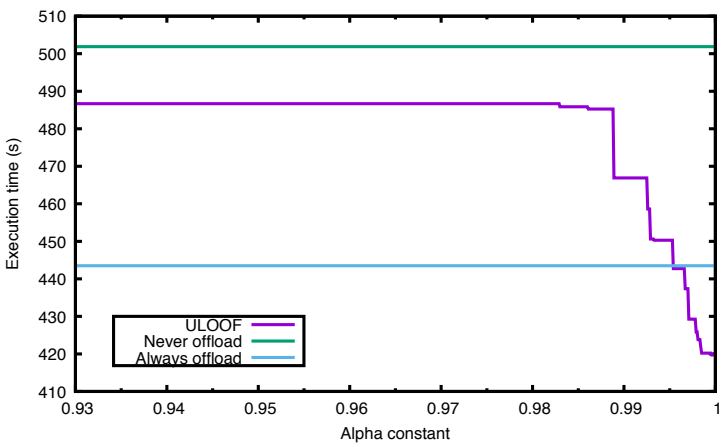

(b) Execution time

Fig. 15. ULOOF performance as a function of $\alpha$ (Fibonacci).

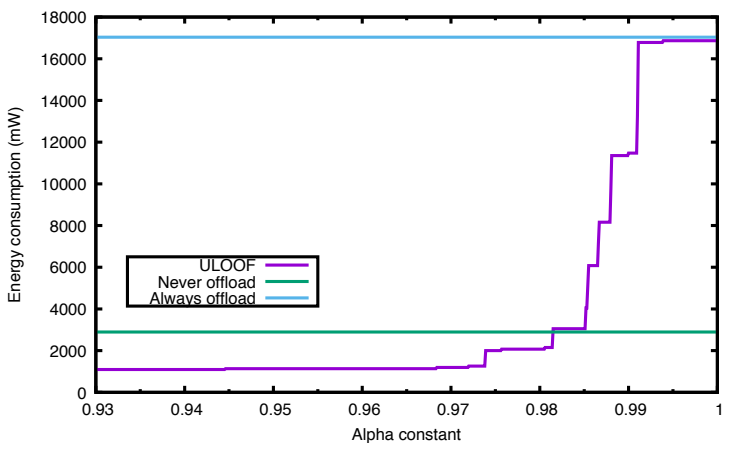

(a) Energy consumption

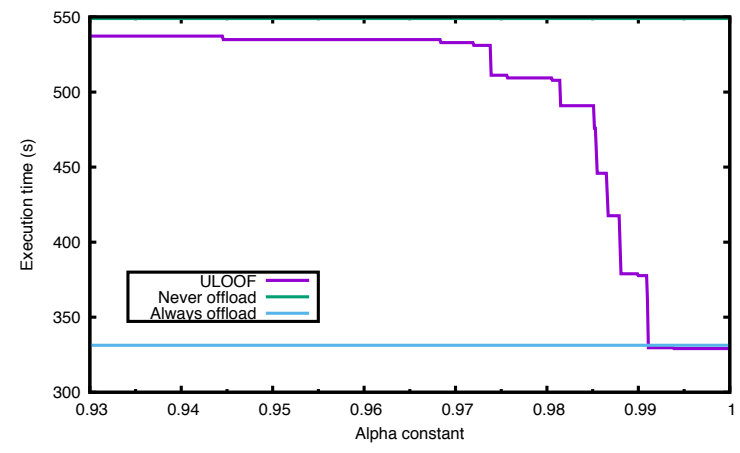

(b) Execution time

Fig. 16. ULOOF performance as a function of $\alpha$ (modified Fibonacci).

efficient compared to the S5 although they have similar application processor (Qualcomm Snapdragon 801 MSM8974-AC @ 2.5GHz and Qualcomm Snapdragon 801 MSM8974 @ 2.3GHz). This may be due to the fact that the Galaxy Note 3 has larger memory (3GB) compared to Galaxy S5 (2GB), handling the route data and map data more efficiently than S5 and in less need of garbage collection of Dalvik VM. The S7 is more than twice faster and energy efficient; this is likely due to the recent processor used in the S7 (Qualcomm Snapdragon 820 MSM8996 @ 2.2GHz), and the higher amount of available live memory (4GB).

\section{Conclusions}

This article presented the ULOOF mobile computation framework, a user-level online computation offloading framework including an innovative decision engine to decrease energy consumption of mobile devices and the execution time of mobile applications. The ULOOF decision engine exploits empirical profiles to predict the energy consumption and execution time of Android application methods, using an assessment of the inputs, and by taking location awareness into account. It uses a low overhead energy consumption model to aid in the mobile offloading decision process. ULOOF does not require any special configuration nor modifications to the runtime of both the device and the edge computing platform, being easily plugged into any framework and application without the need to root or modify the device operating system. An example of ULOOFed application is available in [26].

The framework was evaluated by testbed experiments and large-scale simulations using real data from a major cellular access provider. The results show that both execution time and energy usage can be significantly improved by offloading methods to an external server. We considered both a nearby server (local cloud) scenario, like in MEC environments, and a remote cloud scenario with a longer network latency. The effectiveness of the modelling was evaluated, measuring the accuracy of the interpolations as well as whether the bandwidth actually changes among cell towers. The results indicate that ULOOF can reduce the energy consumption on the mobile device of roughly $50 \%$ for $\mathrm{Wi}$-Fi scenarios with low cloud access latency, and lower yet positive gains also for situations with high latency.

Further work is needed to (i) conceive supervised learning approaches for prediction the mobility behavior of the user and hence improve the ULOOF prediction accuracy, (ii) address prediction challenges for multiple-user single-server situations, i.e., edge computing situations where multiple users may share a single (or a limited number of) offloading server(s), which would make more sense when the driver of the offloaded application is the application provider (doing it in a transparent way with respect to the user) rather than the user itself. We also plan to release in [26] additional bricks of the software framework to allow for reproducibility and enhancements by the community.

\section{ACKNOWLEDGEMENTS}

This work was funded by the CNRS-FAPEMIG WINDS (Systems for Mobile Cloud Computing), ANR ABCD (Adaptive Behavior and Cloud Distribution) and FUI PODIUM (Platform for secure data mobile cloud offloading) projects. We thank C. Ziemlicky for his support with the mobile dataset, and A. Zanni and A. Diamanti for the automated method selection algorithm.

TABLE 2

Comparison between 3 mobile devices (CityRoute application).

\begin{tabular}{|l|l|l|l|l|}
\hline Device & Memory & $\begin{array}{l}\text { CPU } \\
\text { ticks }\end{array}$ & $\begin{array}{l}\text { Execution } \\
\text { time (ms) }\end{array}$ & $\begin{array}{l}\text { Energy consumption } \\
\text { (mW) }\end{array}$ \\
\hline Galaxy S5 & 2 GB & 7196.8 & 73938.84 & 3802104.06 \\
\hline Gal. Note 3 & $3 \mathrm{~GB}$ & 5542.1 & 52347.26 & 2791739.37 \\
\hline Galaxy S7 & $4 \mathrm{~GB}$ & 2602.9 & 21343.0 & 1097507.31 \\
\hline
\end{tabular}




\section{Appendix: Energy Consumption Profiling}

The energy consumption of the CPU and the radio interfaces must be derived empirically for each device. For the CPU, we must derive a function that maps the number of ticks of a method into the energy consumed running that method. Similarly, for the wireless interfaces we must derive a function that maps the number of bytes transmitted into the energy consumption of that data transmission on a certain interface.

Preliminary tests using Android OS primitives showed that the accuracy of the energy estimations on the OS are very low. Hence, we performed hardware-based profiling using an off-theshelf equipment (KCX-017 adapter) that emulates a charger while measuring the power drained by the device.

To obtain the $l_{c p u}$ and $l_{\text {radio }}$ empirical distributions, we created two distinct Android applications to measure separately the energy consumption of the CPU and the consumption of the network interfaces. All the tests were performed in a Samsung S5 with only the profiling application running. For the CPU test the network interfaces were disabled, and for the tests of the network interfaces only one interface is active at a time.

For the energy consumption of the CPU, we generated a constant CPU load by running an application which keeps multiplying random prime numbers using multiple threads. To generate partial loads on all the cores, a short sleep period is set for each thread, calibrated according to each mobile phone. A sleep period of 100 ms every $5^{(\text {load } \bmod 25) / 5}$ iterations was found to work well, where $0 \leq$ load $\leq 100$. We chose the following set of loads for our tests in terms of number of executions: $L=[10,25,35$, $50,60,75,87,100]$. Each load $L_{i}$ was kept for two minutes in order to obtain sufficient amounts of data.

For radio, an application and a web server were developed to exchange traffic. Preliminary tests were first performed by varying the total transfer time, keeping the number of bytes fixed. This showed to be unfruitful and the current barely modified along the experiment, as verified in [19]. This behaviour is expected, since the radio can keep a low power state during small network loads.

A second batch of tests were performed, in which the server creates a delay of 1 millisecond after a certain number of transmitted bytes. This mechanism controls the throughput of the server, and effectively generated a variable energy consumption. A delay of 1 millisecond every [0,300,600, 1200, 2400, 4800, 9600, $19200,38400,76800,153600]$ bytes was used. The duration of the experiment was set to 2 minutes. Using the data gathered from these experiments, we performed a polynomial curve fitting to find the energy profile of the components.

Fig. 17]shows the empirical results for the energy consumption due to CPU usage $\left(l_{c p u}\right)$, with max-min error bars. It shows an upward trend reaching a plateau at the end, as the CPU reaches the full load. The plotted fitting curve, having a coefficient of determination of 0.96605 , is as follows, where $t$ is the number of ticks in the computation:

$$
\begin{aligned}
l_{\text {cpu }}(s)= & +51.422+2.9076 \cdot t^{1}+0.019306 \cdot t^{2} \\
& +6.7841 \cdot 10^{-5} \cdot t^{3}-8.4491 \cdot 10^{-8} \cdot t^{4}
\end{aligned}
$$

Fig. 18 shows the curve of $4 \mathrm{G}$ and Wi-Fi radio interface consumption, as a function of the amount of transferred bytes per second $(b)$. The fitting curves, with a coefficient of determination of 0.99020 and 0.84887 for Wi-Fi and $4 \mathrm{G}$ respectively, are:

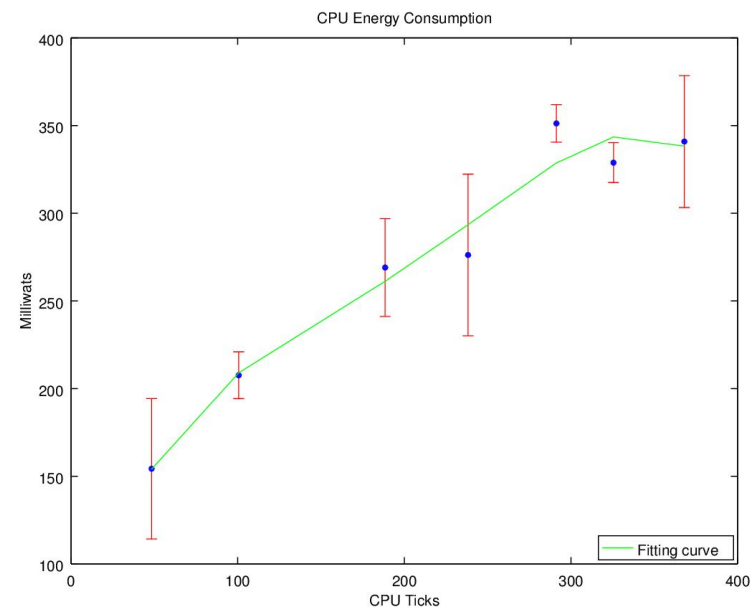

Fig. 17. Power consumption for CPU usage (with standard deviation).

$l_{\text {radio }}(s)=\left\{\begin{array}{l}+111.24-7.9499 \cdot 10^{-5} \cdot b^{1}+1.5999 \cdot 10^{-10} \cdot b^{2} \\ -8.3738 \cdot 10^{-17} \cdot b^{3}+1.3748 \cdot 10^{-23} \cdot b^{4}, \quad \text { if } 4 \mathrm{G} \\ +158.37+1.1811 \cdot 10^{-5} \cdot b^{1}-1.4722 \cdot 10^{-12} \cdot b^{2} \\ +6.1454 \cdot 10^{-20} b^{3}+1.8794 \cdot 10^{-26} b^{4}, \quad \text { if } \mathrm{Wi}-\mathrm{Fi}\end{array}\right.$

\section{REFERENCES}

[1] D. Chaffey, "Mobile marketing statistics 2016," Apr. 2016.

[2] X. Ma, "Characterizing the Performance and Power Consumption of $3 \mathrm{~d}$ Mobile Games," Computer, vol. 46, no. 4, pp. 76-82, Apr. 2013.

[3] Y. Mao, J. Zhang, K. B. Letaief, "Dynamic computation offloading for mobile-edge computing with energy harvesting devices," IEEE J. on Selected Areas in Communications 34(12), 3590-3605, 2016.

[4] R. Kemp et al., "Cuckoo: A Computation Offloading Framework for Smartphones," in Mobile Computing, Applications, and Services, LNCS, Springer Berltin Heidelberg, 2012, vol. 76, pp. 59-79.

[5] E. Cuervo et al., P. Bahl, "MAUI: Making Smartphones Last Longer with Code Offload," in ACM MobiSys 2010.

[6] A. Pamboris, "Mobile Code Offloading for Multiple Resources," Ph.D. dissertation, Imperial College London, 2014.

[7] M. ETSI, "Mobile-Edge Computing," Introductory Technical White Paper, September, 2014.

[8] B.-G. Chun et al., "CloneCloud: Elastic Execution Between Mobile Device and Cloud," in ACM EuroSys 2011.

[9] C. Shi et al., "COSMOS: Computation Offloading As a Service for Mobile Devices," in ACM MobiHoc 2014.

[10] S. Kosta et al., "Thinkair: Dynamic resource allocation and parallel execution in the cloud for mobile code offloading," in IEEE INFOCOM 2012.

[11] J. L. D. Neto, D. F. Macedo, J. M. S. Nogueira, "Location aware decision engine to offload mobile computation to the cloud," in NOMS 2016.

[12] T. Verbelen, P. Simoens, F. D. Turck, B. Dhoedt, "AIOLOS: Middleware for improving mobile application performance through cyber foraging," J. of Systems and Software, vol. 85, no. 11, pp. 2629 - 2639, 2012.

[13] R. Esteves, M. McCool, C. Lemieux, "Real options for mobile communication management," in IEEE GLOBECOM Workshops.

[14] M. Kristensen, "Scavenger: Transparent development of efficient cyber foraging applications," in IEEE PERCOM 2010.

[15] T. L. Cignetti, K. Komarov, C. S. Ellis, "Energy Estimation Tools for the Palm," in ACM MSWIM 2000.

[16] A. Pathak, Y. C. Hu, M. Zhang, "Where is the Energy Spent Inside My App?: Fine Grained Energy Accounting on Smartphones with Eprof," in ACM EuroSys 2012.

[17] S. Hao et al., "Estimating Mobile Application Energy Consumption Using Program Analysis," in IEEE ICSE 2013.

[18] L. Zhang et al., "Accurate online power estimation and automatic battery behavior based power model generation for smartphones," in CODESISSS 2010.

[19] A. P. Miettinen, J. K. Nurminen, "Energy Efficiency of Mobile Clients in Cloud Computing," in USENIX HotCloud 2010.

[20] R. Vallée-Rai et al., "Soot - a Java Bytecode Optimization Framework," in CASCON 1999. 


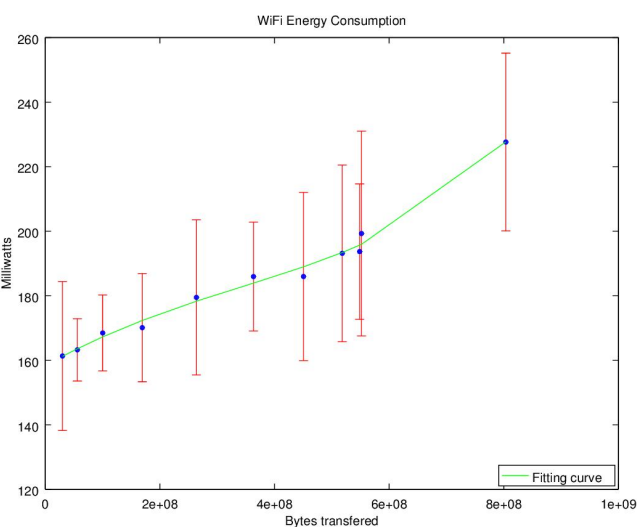

(a) $\mathrm{Wi}-\mathrm{Fi}$

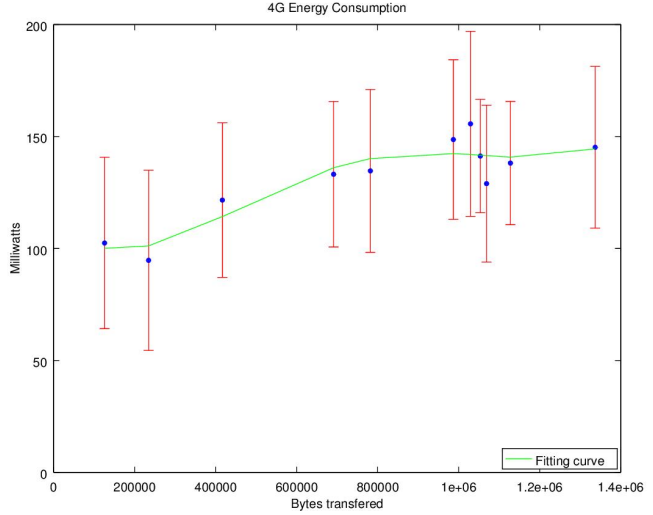

(b) $4 \mathrm{G}$

Fig. 18. Power consumption experimental distribution for different traffic loads and network interfaces.

[21] P. Hudak, "Conception, Evolution, and Application of Functional Programming Languages," ACM Comput. Surv., vol. 21, no. 3, pp. 359-411, Sep. 1989.

[22] H. Akima, "A New Method of Interpolation and Smooth Curve Fitting Based on Local Procedures," J. ACM, vol. 17, no. 4, pp. 589-602, Oct. 1970.

[23] G. Wolberg, I. Alfy, "Monotonic cubic spline interpolation," in Computer Graphics International, 1999. Proceedings.

[24] J. Leskovec, R. Sosič, "SNAP: A General-Purpose Network Analysis and Graph-Mining Library," ACM Trans. on Intelligent Systems and Technology, vol. 8, no. 1, p. 1, 2016.

[25] H. Jiang et al., "Understanding Bufferbloat in Cellular Networks," in ACM SIGCOMM 2012, CellNet Workshop.

[26] ULOOF project website: https://uloof.lip6.fr

[27] Secci, S. , Raad, P. , Gallard, P., "Linking Virtual Machine Mobility to User Mobility", IEEE Trans. on Network and Service Management, Vol. 13, No. 4, pp: 927-940, Dec. 2016.

[28] McCabe, T. J., "A complexity measure," IEEE Transactions on Software Engineering, vol. SE-2, no. 4, pp. 308-320, Dec 1976.

[29] Shepperd, M., "A critique of cyclomatic complexity as a software metric," Software Engineering Journal, vol. 3, no. 2, pp. 30-36, March 1988.

[30] Paxson, V., Allman, M., "Computing TCP's Retransmission Timer," RFC Editor, RFC 2988, Nov. 2000.

[31] Corral, L., et al., "A Method for Characterizing Energy Consumption in Android Smartphones," in GREENS 2013.

[32] A. Zanni, et al., "Automated Selection of Offloadable Tasks for Mobile Computation Offloading in Edge Computing", in CNSM 2017.

[33] Mach, P., and Becvar, Z. "Mobile Edge Computing: A Survey on Architecture and Computation Offloading". IEEE Communications Surveys Tutorials 19, 3 (2017), 1628-1656.

[34] Wang, C., et al., "Computation Offloading and Resource Allocation in Wireless Cellular Networks With Mobile Edge Computing". IEEE Trans. on Wireless Communications 16, 8 (Aug. 2017), 4924-4938.

[35] Wang, C., Yu, F. R., Liang, C., Chen, Q., and Tang, L. "Joint Computation Offloading and Interference Management in Wireless Cellular Networks with Mobile Edge Computing". IEEE Trans. on Vehicular Technology 66, 8 (Aug. 2017), 7432-7445.

[36] Wang, F., Xu, J., Wang, X., and Cui, S. "Joint offloading and computing optimization in wireless powered mobile-edge computing systems". In 2017 IEEE ICC 2017.

[37] Dinh, T. Q., et al., "Offloading in Mobile Edge Computing: Task Allocation and Computational Frequency Scaling". IEEE Trans. on Communications 65, 8 (Aug. 2017), 3571-3584.

[38] Android-x86 - Porting Android to x86. http://www.android-x86.org

[39] Chen, Z., et al., "An Empirical Study of Latency in an Emerging Class of Edge Computing Applications for Wearable Cognitive Assistance". In ACM/IEEE SEC 2017.

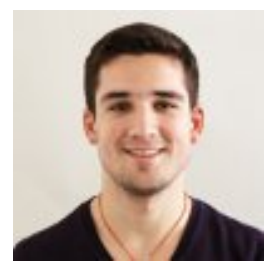

José Leal D. Neto is working as a software engineer at Google Inc, Belo Horizonte, Brazil. $\mathrm{He}$ holds a Msc and undergraduate degree from Univ. Federal of Minas Gerais in 2016, and visited LIP6, France, in 2015-2016.

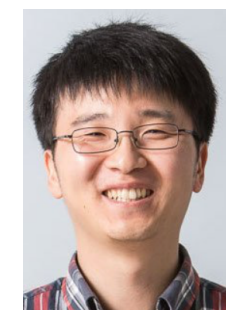

Se-young $\mathbf{Y u}$ is working as postdoctoral researcher at Northwestern University, USA, and was before a postdoc at LIP6 in 2016-2017. He obtained a Ph.D. from University of Auckland, New Zeland.

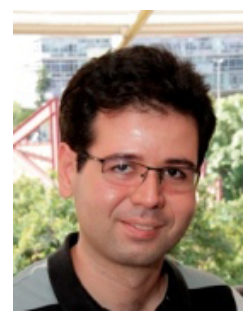

Daniel F. Macedo is a Professor at Univ. Federal of Minas Gerais, Belo Horizonte, Brazil. He obtained a Ph.D. from LIP6, UPMC (now Sorbonne Université), in 2009, and was a visiting professor in the same institution in 2016. His research interests include network management, wireless networks and network programmability. Webpage: http://homepages.dcc. ufmg.br/ damacedo

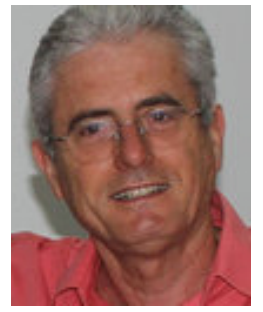

José Marcos S. Nogueira is a Full Professor at Univ. Federal of Minas Gerais, Belo Horizonte, Brazil. He obtained his PhD in Electrical Engineering in UNICAMP, Brazil, in 1985. He was a visiting professor in LIP6, UPMC (now Sorbonne Université), France, in 2016. His research interests include network management, wireless networks, the Internet of Things, as well as mobile, vehicular and opportunistic networks. Webpage: http://homepages.dcc.ufmg.br/ jmarcos

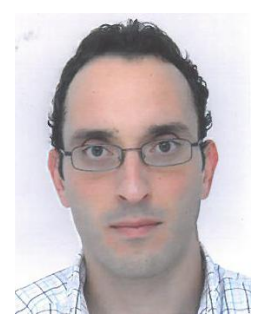

Rami Langar is a Full Professor of Computer Science at University Paris Est Marne-la-Vallée, France. From 2008 to 2016, he was Associate Professor at LIP6, UPMC (now Sorbonne Université), France. He obtained his Ph.D. from Telecom ParisTech, France, in 2006. His research interests include resource management in future wireless systems, cloud-RAN, Software Defined Wireless Networks, and Mobile Edge Cloud. Webpage: http://perso.u-pem.fr/ langar

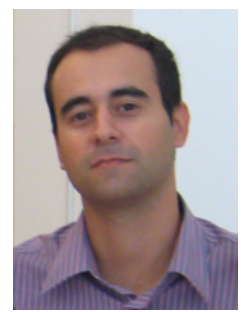

Stefano Secci is an Associate Professor at the LIP6, Sorbonne Université, Paris, France, since 2010. He obtained a dual Ph.D. in 2009 from Politecnico di Milano, Italy, and Telecom ParisTech, France. He is active in the areas of network resource allocation, network optimization and analytics, virtualization, Internet protocol design and experimentation. Webpage: https://lip6. fr/Stefano.Secci 